APS

physics

This is the accepted manuscript made available via CHORUS, the article has been published as:

\title{
Direct Observation of Resonant Scattering Phase Shifts and Their Energy Dependence
}

Stephen D. Gensemer, Ross B. Martin-Wells, Aaron W. Bennett, and Kurt Gibble Phys. Rev. Lett. 109, 263201 - Published 26 December 2012 DOI: 10.1103/PhysRevLett.109.263201 


\title{
Direct Observation of Resonant Scattering Phase Shifts and their Energy Dependence
}

\author{
Stephen D. Gensemer, ${ }^{1}$ Ross B. Martin-Wells, Aaron W. Bennett, \& Kurt Gibble \\ Department of Physics, The Pennsylvania State University, University Park, PA 16802, USA
}

We scan the collision energy of two clouds of cesium atoms between 12 and $50 \mu \mathrm{K}$ in atomic fountain clock. By directly detecting the difference of s-wave scattering phase shifts, we observe a rapid variation of a scattering phase shift through a series of Feshbach resonances. At the energies we use, resonances that overlap at threshold become resolved. Our statistical phase uncertainty of $8 \mathrm{mrad}$ can be improved in future precision measurements of Feshbach resonances to accurately determine the Cs-Cs interactions, which may provide stringent limits on the time variation of fundamental constants.

PACS: 34.50.Cx, $\rho 06.30 . \mathrm{Ft}$

Feshbach scattering resonances occur when the continuum state of two colliding particles couples to a bound state (Fig. 1) [1]. Feshbach resonances have found wide applicability in dilute, ultracold, atomic and molecular gases because they provide an accessible control of the inter-particle interactions [2-6]. Feshbach's elegant treatment of scattering resonances showed that scattering phase shifts, and hence cross sections, change rapidly as the collision energy tunes through resonance. The rapid phase change is a general feature of resonance phenomena and the resonant energy dependence of cross sections has been observed in a variety of experiments, including neutron and electron scattering and photodetachment [7-9]. In ultracold gases, so far magnetic fields have been used to tune resonances to threshold, changing the energy of the bound state by vertically translating the grey potential in Fig. 1, instead of tuning the collision ${ }^{1}$ Present address: Centre for Engineered Quantum Systems, School of Physics, The University of Sydney, NSW 2006 Australia. 
energy [3-11]. Here, we scan the collision energy between two ultracold clouds of cesium atoms in an atomic clock and directly observe the scattering phase shift [12] through a series of scattering resonances. Increasing the collision energy allows us to resolve resonances that overlap at threshold. Precise measurements of scattering phase shifts through a resonance will very accurately determine the resonance position, giving a highly precise determination of the atomic interactions [5] and a potential route to stringent limits on the time variation of fundamental constants $[13,14]$.

We directly measure scattering phase shifts by preparing cesium atoms in coherent superpositions of the two clock states and detecting the phase shift of these coherences after the clock atoms scatter off atoms prepared in a pure 'target' state (Fig. 2 inset) [12]. When the $|40\rangle$ (| 30)) clock state scatters off the target atoms, it acquires a scattering phase shift $\delta_{4}\left(\delta_{3}\right)$. The phase of the clock coherence, the superposition of $\left|\mathrm{F}=3, \mathrm{~m}_{\mathrm{F}}=0\right\rangle$ and $|40\rangle$, precesses as hands on a clock. The scattering causes the phase of the coherence to jump by the difference of the scattering phase shifts, $\Phi=\delta_{4}-\delta_{3}$ [12], represented by the time difference between the ring of scattered clocks and the unscattered clock in the Fig. 2 inset. We directly detect $\Phi$ as a phase shift of the clock's Ramsey fringes as in Fig. 2(b). To be sensitive to the s-wave phase shifts, we detect atoms that scatter near $90^{\circ}$, using the Doppler shift of a stimulated-Raman transition $[12,15]$. By also measuring the cross sections for each clock state on $|32\rangle$, we isolate the scattering resonances to only the $|32\rangle \otimes|40\rangle$ channel, and exclude the $|32\rangle \otimes|30\rangle$ channel.

In our fountain clock, we launch two clouds of cesium atoms with short time delays, 6.5-11 $\mathrm{ms}$, to give collision energies between 12 and $50 \mu \mathrm{K}[12,16]$. A series of microwave pulses in three microwave cavities state prepares the atoms in the first cloud (C1) in a desired $\left|3, \mathrm{~m}_{\mathrm{F}}=1,2,3\right\rangle$ target state and the second cloud (C2) in $|41\rangle$. A two-photon stimulated Raman transition transfers a $270 \mathrm{nK}$ wide slice of the $\mathrm{C} 2$ vertical velocity distribution from $|41\rangle$ to $|30\rangle$. Interleaved with these transitions are clearing laser pulses tuned to the $6 \mathrm{~s}_{1 / 2}, \mathrm{~F}=4 \rightarrow 6 \mathrm{p}_{3 / 2}, \mathrm{~F}^{\prime}=5$ ' and $2-3$ ' transitions that remove unselected atoms. The microwave clock cavity then prepares $\mathrm{C} 2$ 
atoms in a coherent superposition of $|30\rangle$ and $|40\rangle$ and the clouds collide near the apogee. A small fraction of the atoms in the clouds scatter, forming an expanding spherical shell of atoms, whose coherence is shifted by the difference of their s-wave scattering phase shifts. When the scattered shell is centered in the clock cavity, a second $\pi / 2$ clock pulse converts the phase of the scattered atoms' coherence into a population difference between $|30\rangle$ and $|40\rangle$. A clearing pulse then removes the $\mathrm{C} 1$ target atoms and the atoms in $|30\rangle$. To detect the scattered atom's Ramsey fringe, we velocity-selectively transfer atoms that scatter near $90^{\circ}$ from $|40\rangle$ to $|30\rangle$. After clearing and optical pumping pulses remove $\mathrm{F}=4$ atoms, a laser beam tuned to $\mathrm{F}=4 \rightarrow \mathrm{F}^{\prime}=5$ excites the atoms and we collect the fluorescence with a lens and photodiode. To detect the phase shift of the scattered atoms, we use the phase of the unscattered atoms as a reference (Fig. 2(B)) and, to measure and remove backgrounds, we use a pump-probe technique where we inhibit the selection of either $\mathrm{C} 1, \mathrm{C} 2$, and both clouds [12].

Fig. 2 shows a large and rapid variation of the scattering phase shifts $\Phi$ as a function of collision energy $E_{c}$ and magnetic field $B$ for target atoms prepared in $|32\rangle$. Feshbach resonances generally tune with both the collision energy $E_{c}$ and magnetic field $B$, with a slope in the $E_{c}-B$ plane that is given by the difference of the magnetic moments of the coupled bound state and the incident channel. The black lines in Fig. 2 show the slopes for magnetic moments of 1/2, 1, 3/2, and 2 Bohr magnetons, $\mu_{\mathrm{B}}$. Strikingly, all of the features we observe for $|32\rangle$ target atoms, as well as $|33\rangle$ and $|31\rangle$ targets below, follow one of these slopes with intercepts that are near the origin of the $\mathrm{E}_{\mathrm{c}}$ - $\mathrm{B}$ plane. For a single narrow Feshbach resonance, the observed width would be proportional to the spread of collision energies, roughly proportional to $E_{c}^{1 / 2}$. Instead, the features in Fig. 2 follow the black lines, scaling linearly with energy, as expected if there were two (or more) Feshbach resonances, with different magnetic moments. The small intercepts of the features suggest that the bound states have small binding energies (at $\mathrm{B}=0$ ), lying very close to threshold, $E_{c}=0$. Further, if the slopes are multiples of $\mu_{\mathrm{B}} / 2$, it is likely that the bound states are halo molecular states, which have magnetic moments that are essentially the same as those for 
free atoms. For s-wave Feshbach resonances, the halo states asymptotically correspond to $|31\rangle \otimes$ $|41\rangle,|30\rangle \otimes|42\rangle,|3,-1\rangle \otimes|43\rangle$, and $|3,-2\rangle \otimes|44\rangle$ at large internuclear separations. The magnetic moments for these halo states near threshold give resonance positions near the black lines in Fig. 2. We note that coupling to these states conserves the total $\mathrm{z}$ angular momentum, the total molecular $\mathrm{M}_{\mathrm{F}}$, as required for an s-wave resonance. If the Feshbach resonances had an angular momentum higher than s-wave, other total $\mathrm{M}_{\mathrm{F}}$ 's would be allowed, with corresponding differences of magnetic moments. We have not observed these for target atoms in $|32\rangle$, or $|31\rangle$ or $|33\rangle$.

The shape of the resonant phase shifts in Fig. 2(a) versus magnetic field is remarkably similar for all of our collision energies $\mathrm{E}_{\mathrm{c}}$. If the corresponding bound states for all the Feshbach resonances have the same binding energy $\Delta \mathrm{E} \approx 0$, scaling the magnetic field $\mathrm{B}$ as $\mathrm{B} /\left(\mathrm{E}_{\mathrm{c}}+\Delta \mathrm{E}\right)$ should align all of the resonances, as in Fig. 2(c). As for any general resonance, the scattering phase shift goes through $\pi$ as we sweep across a resonance. However, if each resonance is narrower than our rms spread of collision energies $\delta \mathrm{E}$, the peak-to-peak phase excursions will be smaller, with an amplitude proportional to $\delta \mathrm{E}^{-1}$. We prepare the atoms in each cloud with a mean temperature of $590 \mathrm{nK}$, which gives $\delta \mathrm{E}=3.2 \mu \mathrm{K}$ at $\mathrm{E}_{\mathrm{c}}=12.2 \mu \mathrm{K}$ and a larger $\delta \mathrm{E}=8.9 \mu \mathrm{K}$ at $49.2 \mu \mathrm{K}$, proportional to $\mathrm{E}_{\mathrm{c}}^{1 / 2}[16]$. Thus, the observed peak-to-peak phase excursions are smaller for large $\mathrm{E}_{\mathrm{c}}$. With this phase and $\mathrm{B} / \mathrm{E}_{\mathrm{c}}$ scaling, the magnetic field dependence through the series of the Feshbach resonances in Fig. 2(c) has nearly the same form over our range of energies. Averaging this scaled data gives a function (black curve) to compare to the measured phase versus $\mathrm{E}_{\mathrm{c}}$ and $\mathrm{B}$. Interestingly, the black curve and the data for each $\mathrm{E}_{\mathrm{c}}$ show the statistical significance of a nearly flat region between $3 / 2$ and $1 \mu_{\mathrm{B}}$; the phase steeply decreases, flattens, and then continues steeply down again for all of the collision energies, which can occur if there are resonances near $3 / 2$ and $1 \mu_{\mathrm{B}}$. We speculate that a Feshbach resonance should also exist near $1 / 2 \mu_{\mathrm{B}}$. If it does, it is apparently sufficiently narrow that we do not resolve it for $|32\rangle$ targets, but 
the bound state coupling is evidently larger for $|33\rangle$ target atoms as we observe this resonance below.

Figure 3(a) depicts the bound state energies versus magnetic field (black lines), for multiples of $\mu_{\mathrm{B}} / 2$ with $\Delta \mathrm{E}=0$. The intersections of these bound state energies with our six chosen collision energies (horizontal lines) represent the positions of potential Feshbach resonances. In Fig. 3(c) the scattering phase shift difference for the $|32\rangle$ target atoms is shown for each $\mathrm{E}_{\mathrm{c}}$ versus $\mathrm{B}$, along with the grey curve, the average of the scaled data in Fig. 2(c). While this curve describes the data quite well, there are deviations that vary systematically with energy. These deviations could be due to the multiple bound states having significantly different binding energies $\Delta \mathrm{E}$. In addition, biases in the effective collision energy can occur since the $|32\rangle \otimes|40\rangle$ and $|32\rangle \otimes|30\rangle$ scattering cross sections are energy dependent. Both contribute to an energy bias of the scattered atoms that we detect. Full coupled-channels calculations yield both the phase and cross-section variations and a comparison of these with such measurements can precisely test and improve our knowledge of the Cs-Cs molecular interactions. We find that all the resonances, when extrapolated to $\mathrm{B}=0$, are consistent with binding energies between 0 and $10 \mu \mathrm{K}$. This is consistent with a previous observation of frequency shift cross sections at threshold, which appeared to indicate the existence of one or two overlapping Feshbach resonances of states with binding energies of $\Delta \mathrm{E}=0<0.5 \mu \mathrm{K}$ at $\mathrm{B}=0[17,18]$.

Figures 3(b) and (d) show the measured phase shift differences for target atoms in $|33\rangle$ and $|31\rangle$. The $|33\rangle$ and $|31\rangle$ scattering shows features near the magnetic moments of the same halo molecular states as $|32\rangle$. Here, $|33\rangle$ scattering has a clear resonance that asymptotically corresponds to $\approx \mu_{\mathrm{B}} / 2$ for the $|32\rangle \otimes|41\rangle$ exit channel.

Fig. 3(e) shows the phase difference for a $|3,-3\rangle$ target, which has no obvious resonances. We use this channel to measure small magnetic field gradients throughout the atomic trajectories in our fountain clock [16], which make a clock precision of 8 mrad challenging at high magnetic fields. While the clock transition, $|30\rangle \rightarrow|40\rangle$, has no linear Zeeman shift, it has a quadratic 
Zeeman shift of $427 \mathrm{~Hz} / \mathrm{G}^{2}$. At $\mathrm{B}=1.8 \mathrm{G}$, the quadratic Zeeman shift is $1.4 \mathrm{kHz}$. With a typical Ramsey fringe linewidth of $4.4 \mathrm{~Hz}$ for $33.3 \mu \mathrm{K}$, an $8 \mathrm{mrad}$ phase uncertainty corresponds to a frequency uncertainty of $11 \mathrm{mHz}$, and a magnetic field uncertainty of $7 \mu \mathrm{G}$. Since we sweep over a wide range of magnetic fields, our clock has no magnetic shielding. To achieve this precision, a flux-gate magnetometer and control system actively stabilizes the vertical field, reducing the background fluctuations of approximately $3 \mathrm{mG}$. We use the unscattered clock atoms [Fig. 2 inset and (b)] to measure the quadratic Zeeman shift along the fountain trajectory. Because the swave halo of scattered atoms follows a different trajectory than the unscattered atoms, magnetic field variations in our clock produce a phase shift of order $100 \mathrm{mrad}$ for $\mathrm{B}>1 \mathrm{G}$. We therefore interleave measurements of $|44\rangle$ or $|3,-3\rangle$ target atoms, prepared with a non-adiabatic magnetic field reversal (Fig. 3(e)). Since the phase shifts for these states show no scattering resonances, we use $|3,-3\rangle$ and $|44\rangle$ targets to measure and correct the magnetic field variations for the scattered halo for other target states [16].

We note that scattering phase shifts have sharp steps as inelastic scattering channels close. While scattering phase shifts wrap through $\pi$ at Feshbach resonances, phase steps at inelastic thresholds do not, and are often small $[19,20]$. The phase excursions we observe are large, even after thermally averaging. Therefore, these are almost certainly Feshbach resonances, along with smaller contributions from inelastic thresholds. The peak-to-peak phase excursions we see are consistent with Feshbach widths of order of several mG. If the widths were significantly wider (narrower), our spread of collision energies would yield larger (smaller) peak-to-peak phase excursions.

The spread of collision energies for two colliding clouds is significantly larger than their temperatures of each cloud for high collision energies. The spread is $\delta \mathrm{E}=\left[\mathrm{E}_{0}\left(\mathrm{~T}_{1}+\mathrm{T}_{2}\right)+3 / 8\left(\mathrm{~T}_{1}+\mathrm{T}_{2}\right)^{2}\right]^{1 / 2}$, where $\mathrm{E}_{0}$ is the collision energy due to the relative velocities of the two clouds ( $T=0$ limit) and $T_{1}$ and $T_{2}$ are the temperatures of each cloud [16]. In our fountain clock, the atoms are cooled using degenerate sideband cooling in a moving-frame optical lattice 
[21]. The first cloud's temperature $(\approx 790 \mathrm{nK})$ is higher than the second's $(\approx 390 \mathrm{nK})$ since the first cloud, multiply loaded in a double-MOT [12], has more atoms than the second, which is launched directly from a vapor-cell MOT.

In conclusion, we directly observe a rapid variation of s-wave scattering phase shifts as we scan the collision energy through a series of Feshbach resonances. Reducing the spread of collision energies could yield even more precise measurements of the phase shifts and the positions of the Feshbach resonances. To resolve these overlapping resonances, the collision energy has to be of order $10 \mu \mathrm{K}$. Currently, our state-preparation velocity selects the second cloud to $270 \mathrm{nK}$ and this velocity selection can be narrower. Adding a narrow velocity selection of the first cloud will help even more. Since only the velocity spread along the collision axis contributes significantly to the spread of collision energies, cooling in one dimension is sufficient [22]. Alternatively, a sample can be evaporatively cooled, split, and then accelerated to collide $[23,24]$. Higher energy resolution and a thorough evaluation of systematic errors will lead to a highly precise determination of the cesium interactions $[5,25,26]$. In turn, these can place stringent limits on the time variations of underlying fundamental forces [13].

We acknowledge discussions with S. Kokkelmans and E. Tiesinga and financial support from the NSF and Penn State. 
[1] H. Feshbach, Annals of Physics 19, 287 (1962).

[2] E. Tiesinga, A. J. Moerdijk, B. J. Verhaar, H. T. C. Stoof, Phys. Rev. A 46, R1167 (1992).

[3] S. Inouye, M. R. Andrews, J. Stenger, H.-J. Miesner, D. M. Stamper-Kurn \& W. Ketterle, Nature 392, 151 (1998).

[4] J. L. Roberts, N. R. Claussen, S. L. Cornish, E. A. Donley, E. A. Cornell, and C. E. Wieman, Phys. Rev. Lett. 86, 4211 (2001).

[5] C. Chin, V. Vuletić, A. J. Kerman, S. Chu, E. Tiesinga, P. J. Leo, and C. J. Williams, Phys. Rev. A 70, 032701 (2004).

[6] C. Chin, R. Grimm, P. Julienne, E. Tiesinga, Rev. Mod. Phys. 82, 1225 (2010).

[7] R. E. Peterson, H. H. Barschall, C. K. Bockelman, Phys. Rev. 79, 593 (1950).

[8] D. Spence, T. Noguchi, Journ. Chem. Phys. 63, 505 (1975).

[9] H. C. Bryant, B. D. Dieterle, J. Donahue, H. Sharifian, H. Tootoonchi, D. M. Wolfe, P. A. M. Gram and M. A. Yates-Williams, Phys. Rev. Lett. 38, 228 (1977).

[10] E. L. Hazlett, Y. Zhang, R. W. Stites, K. M. O’Hara, Phys. Rev. Lett. 108, 045304 (2011).

[11] C. Kohstall, M. Zaccanti, M. Jag, A. Trenkwalder, P. Massignan, G. M. Bruun, F. Schreck \& R. Grimm, Nature 485, 615 (2012).

[12] R. A. Hart, X. Xu, R. Legere, K. Gibble, Nature 446, 892 (2007).

[13] C. Chin, V. V. Flambaum, Phys. Rev. Lett. 96, 230801 (2006

[14] A. Borschevsky, K. Beloy, V. V. Flambaum, and P. Schwerdtfeger, Phys. Rev. A 83, $052706(2011)$.

[15] M. Kasevich, D. S. Weiss, E. Riis, K. Moler, S. Kasapi, and S. Chu, Phys. Rev. Lett. 66, 2297 (1991).

[16] See Supplemental Material at [URL to be inserted by publisher] for more information. 
[17] H. Marion, S. Bize, L. Cacciapuoti, D. Chambon, F. Pereira dos Santos, G. Santarelli, P. Wolf, A. Clairon, A. Luiten M. Tobar, S. Kokkelmans, and C. Salomon, arXiv:physics/0407064 (2004).

[18] D. J. Papoular, S. Bize, A. Clairon, H. Marion, S. J. J. M. F. Kokkelmans, and G. V. Shlyapnikov, Phys. Rev. A 86, 040701R (2012).

[19] S. J. J. M. F. Kokkelmans (private communication).

[20] E. Tiesinga (private communication).

[21] P. Treutlein, K. Y. Chung, S. Chu, Phys. Rev. A 63, 051401 (2001).

[22] H. J. Lee, C. S. Adams, M. Kasevich, S. Chu, Phys. Rev. Lett. 76, 2658 (1996).

[23] N. R. Thomas, Niels Kjærgaard, P. S. Julienne, A. C. Wilson, Phys. Rev. Lett. 93, 173201 (2004).

[24] C. Buggle, J. Léonard, W. von Klitzing, J. T. M. Walraven, Phys. Rev. Lett. 93, 173202 (2004).

[25] K. Szymaniec, W. Chałupczak, E. Tiesinga, C. J. Williams, S. Weyers, and R. Wynands, Phys. Rev. Lett. 98, 153002 (2007),

[26] P. Laurent et al., Appl. Phys. B 84, 683 (2006) 
Fig. 1 (color online). A Feshbach scattering resonance occurs when the collision energy $\mathrm{E}_{\mathrm{c}}$ (aqua solid arrow) has the same energy as a bound state of the two particles. We scan the collision energy from 12 to $49 \mu \mathrm{K}$ (dashed arrows) and observe a series of Cs Cs-scattering resonances. The schematic grey potential energy surface, as a function of internuclear distance R, can also be shifted vertically by changing the magnetic field B, which instead shifts the energy of the bound state $\Delta \mathrm{E}$. Throughout the paper we use energy units of $\mathrm{E}_{\mathrm{c}} / \mathrm{k}_{\mathrm{B}}$.

Fig. 2 (color online). (Inset) Clock atoms in a coherent superposition of two internal clock states scatter off of targets atoms (in $|32\rangle$ ), giving each clock state a quantum scattering phase shift. The phase of the clock coherence is represented by the time of the scattered and unscattered clocks. For scattered atoms, the clock phase is shifted by the difference of the quantum scattering phase shifts $\Phi$ of the two clock states. (a) Resonances of quantum scattering phase shifts versus collision energy $\mathrm{E}_{\mathrm{c}}$ and magnetic field $\mathrm{B}$. The measured phase differences $\Phi$ (grey dots) are the difference of s-wave phase shifts for the cesium clock states scattering off cesium atoms prepared in $|32\rangle$. The black lines illustrate the allowed differences of magnetic moments for an $1=0$ halo molecular state. Features at $\mu_{\mathrm{B}}$ and $3 / 2 \mu_{\mathrm{B}}$ suggest the bound-states are resonant near $\mathrm{E}_{\mathrm{c}}=0$. For clarity, the vertical scale varies linearly with $\mathrm{E}_{\mathrm{c}}$. (b) Atomic clock transition probability with two $\pi / 2$ pulses for scattered (solid) and unscattered (dashed) atoms at $\mathrm{E}_{\mathrm{c}}=12.2 \mu$ $\mathrm{K}$ and $\mathrm{B}=0.3 \mathrm{G}$. The phase shift $\Phi$ is plotted in (a). Each point represents the average of 18 fountain launch measurements, plus background measurements [16]. (c) $\Phi$ from (a) versus B, scaled by $E_{c}$, yields essentially the same shape for all $E_{c}$, as expected for the peculiar case of bound states near threshold. Here the amplitude of $\Phi$ is scaled by the spread of collision energies $\delta \mathrm{E}$ as discussed in the text. The black vertical lines represent the same magnetic moments in (a) and the data point colors are from Fig. 1. The weighted average of $\Phi_{\text {sc }}$ versus B gives an approximate reference shape for all energies (grey curves). 
Fig. 3 (color online). (a) Positions of Feshbach scattering resonances versus $\mathrm{E}_{\mathrm{c}}$ and $\mathrm{B}$, denoted by the large symbols (squares, diamonds, circles, triangles), for molecular bound states that are resonant at threshold, with magnetic moments that are different from those for bare atoms by $(1 / 2$, $1,3 / 2,2) \mu_{\mathrm{B}}$. The experimental $\mathrm{E}_{\mathrm{c}}$ 's are depicted by the dashed horizontal lines. (b-e) Measured difference of s-wave quantum scattering phase shifts for 6 collision energies $E_{c}$ versus magnetic field B, for target atoms in $|33\rangle,|32\rangle,|31\rangle$, and $|3,-3\rangle$. As in Fig. 2(a), the scattering resonances shift with $E_{c}$ and $B$. In (b-d) at all energies, scattering resonances occur near the symbols from (a) (see text). (c) Inset: The scattering cross section of $|32\rangle$ on $|40\rangle$ (magenta) shows scattering resonances, whereas $|32\rangle$ on $|30\rangle$ does not. In (e), note that the target for $12.2 \mu \mathrm{K}$ is $|44\rangle$. 


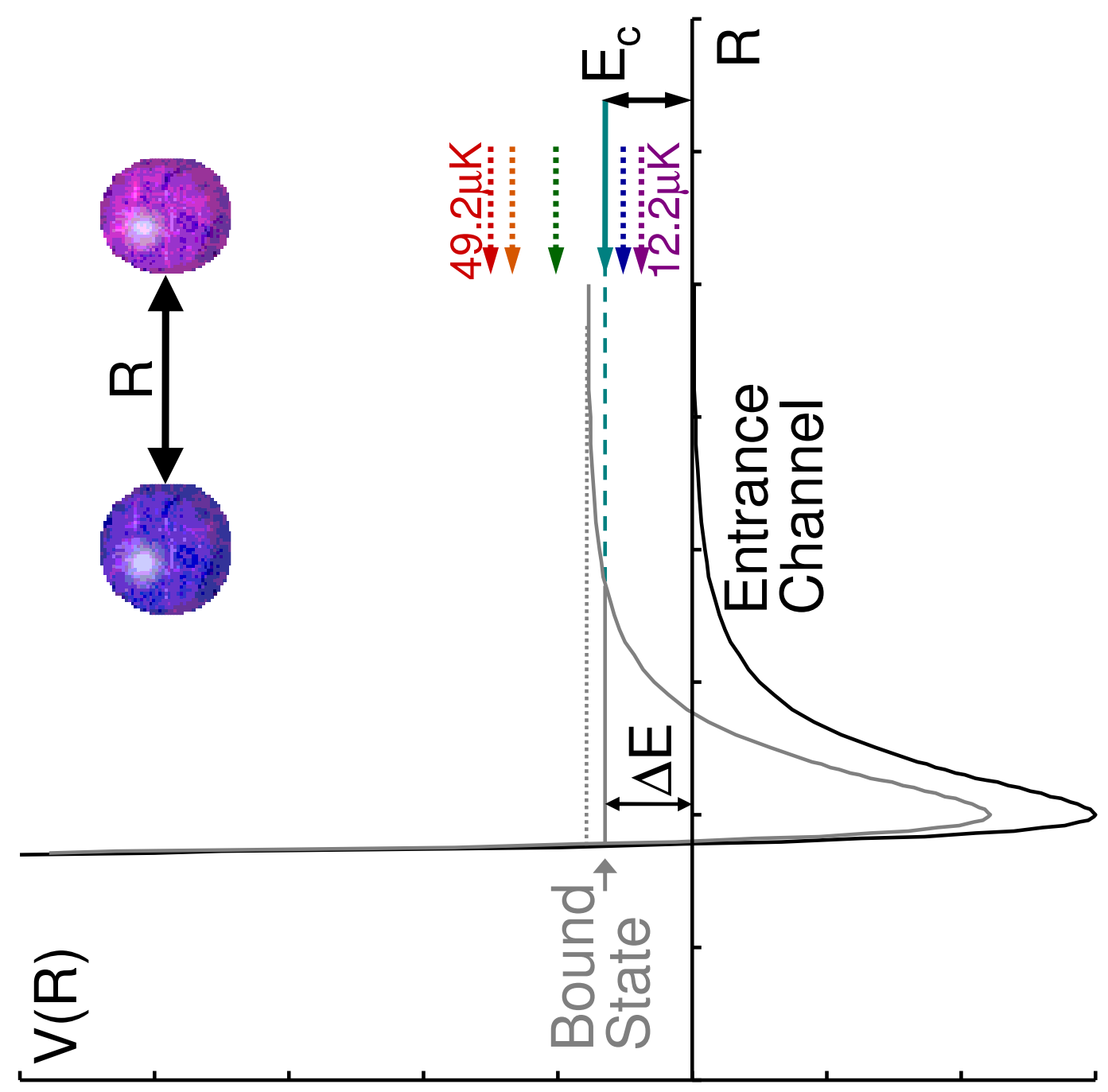

㐫

Figure $1 \quad$ LW13383 19 NOV2012 


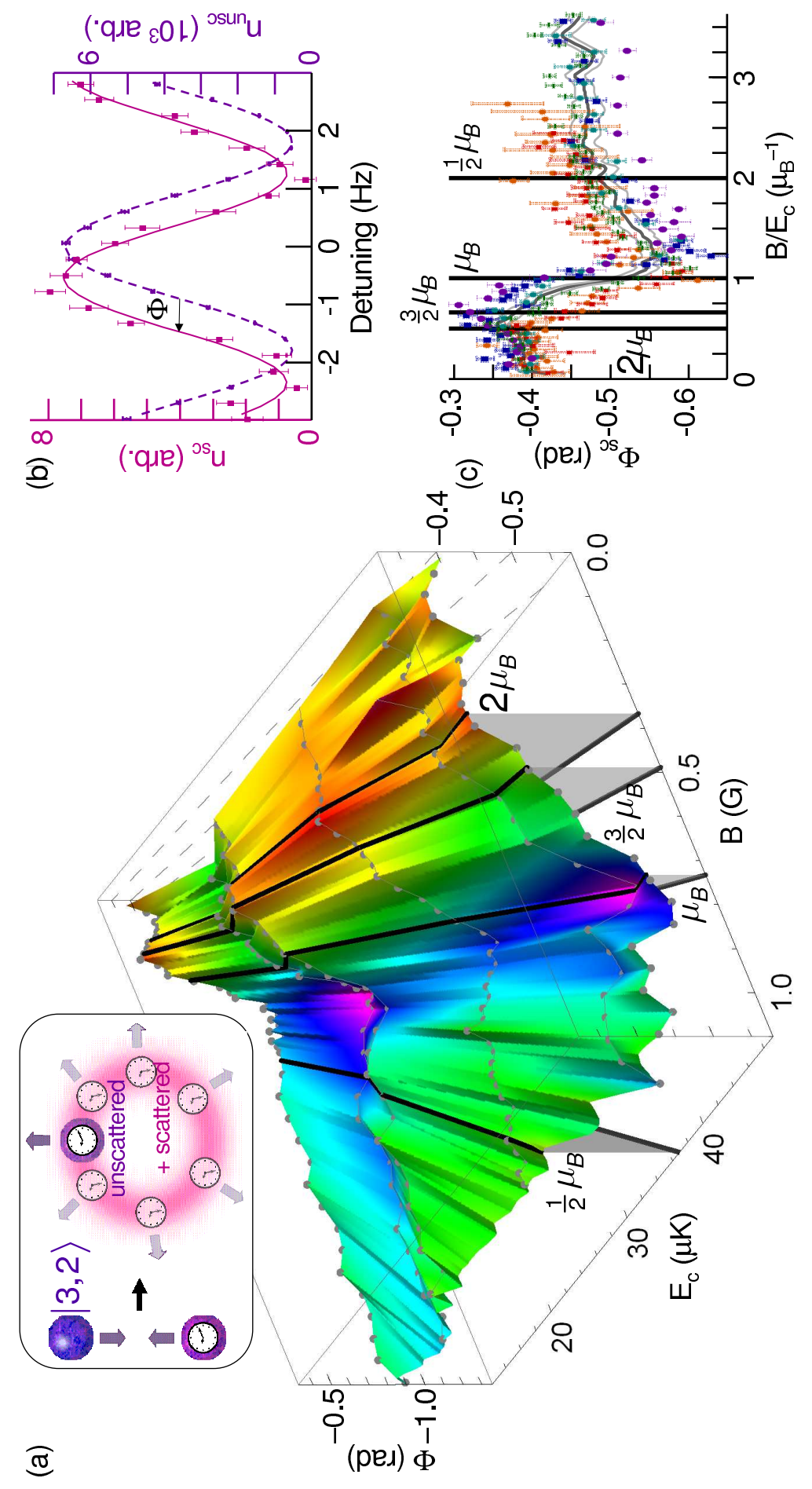

Figure 2 LW13383 19NOV2012 

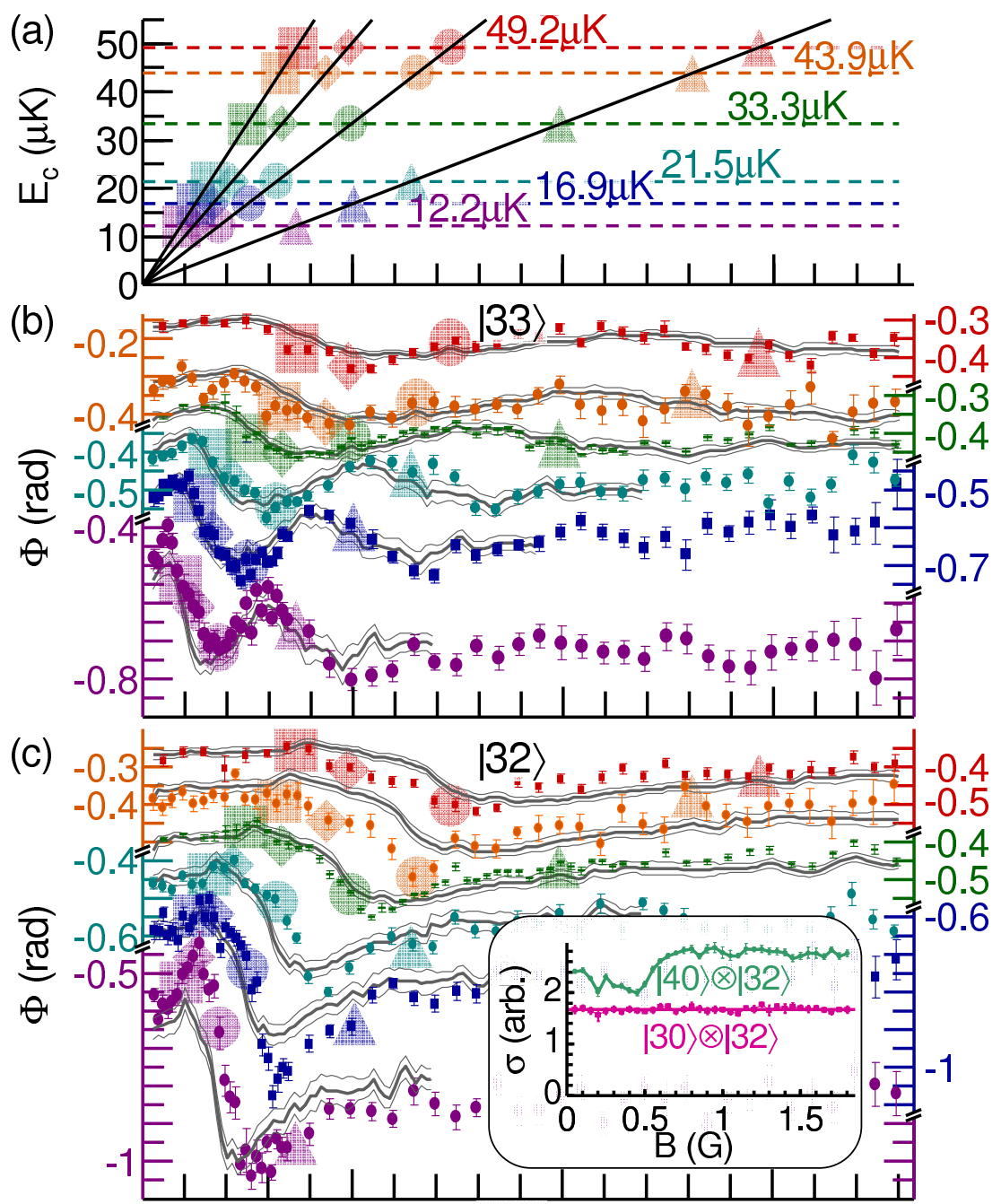

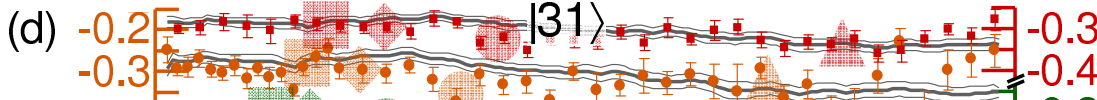
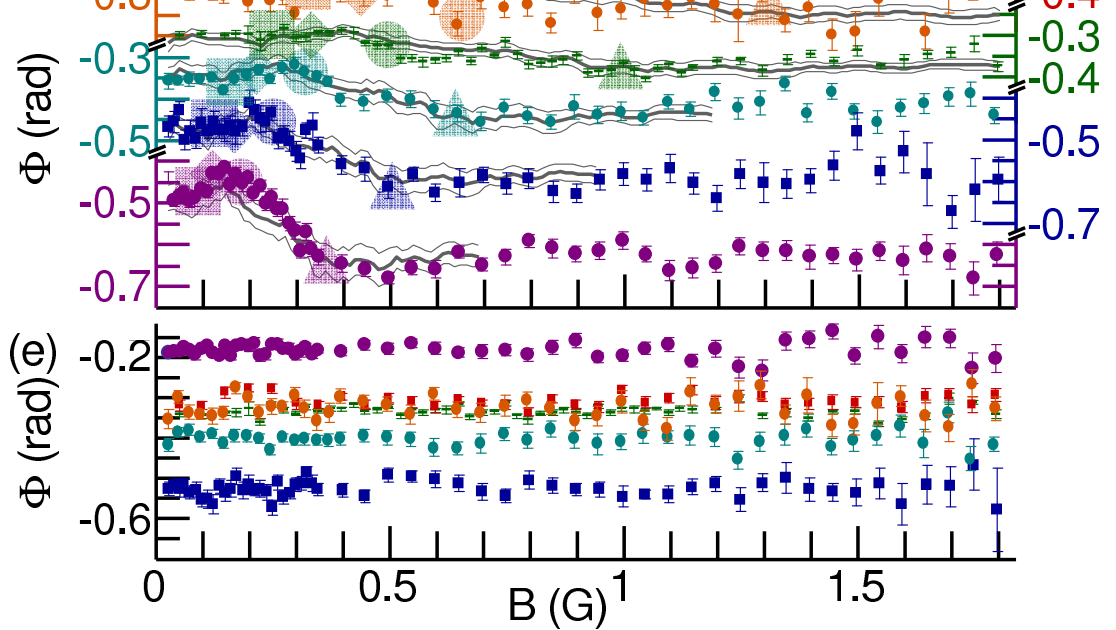